\title{
Monoclonal Gammopathy of Undetermined Significance
}

National Cancer Institute

\section{Source}

National Cancer Institute. Monoclonal Gammopathy of Undetermined Significance. NCI

Thesaurus. Code C3996.

A condition in which an abnormal amount of a single immunoglobulin is present in the serum. This category includes IgM monoclonal gammopathy of undetermined significance and non-IgM monoclonal gammopathy of undetermined significance. Up to $25 \%$ of cases of monoclonal gammopathy of undetermined sig nificance progress to a B-cell malignancy or myeloma. 